\title{
活细胞内细胞器转动理论
}

\author{
唐孝 威 \\ (中国科学院高能物理研究所, 北京 100039)
}

\section{关组词 细胞器转动、ATP分子水解、布朗转动}

细胞器运动是活细胞内部普遍存在的现象 ${ }^{[1]}$. 除细胞器平移运动外, 文献上报道讨细胞 器作为整体的转动 ${ }^{23}$. 已经知道, 细胞器由同它结合的运动蛋白驱动, 由 ATP 分子水解提供 能量, 沿着微丝或微管轨道运动 ${ }^{[3-s]}$. 细胞器沿直线轨道的运动, 表现为细胞器的平移运动. 而细胞器沿轨道的滚动 ${ }^{[6,}$, 以及沿曲率半径很小的弯曲轨道的转折运动, 表现为细胞器绕自身 轴的转动. 我们曾定量讨论过细胞器的平移运动的, 本文提出细胞器转动的公式, 并且定量地 讨论细胞器转动与由分子热运动引起的布朗转动的区别.

\section{1 细胞器转动公式}

设球形细胞器半径为 $a$, 悬浮在粘度为 $\eta$ 的细胞质中, 根据粘性流体中球体缓慢转动的: 理论 ${ }^{[8]}$, 当有旋转力矩 $D$ 作用在细胞器上时, 细胞器在细胞质中以角速度 $\omega$ 绕自身轴转动, 角 速度和旋转力矩的关系是

$$
D=8 \pi \eta a^{3} \omega .
$$

若细胞器在持续时间 6 内转动, 且角速度保持恒定,细胞器在这段时间内的转动角度

$$
\theta-\omega t,
$$

旋转力矩对细胞器所作的功是 $D \theta$, 相应的能量 $E$ 由 ATP 分子水解来提供,因而

$$
E-D \theta \text {. }
$$

由 ATP 分子水解提供的驱动功率

$$
P=E / l,
$$

$P$ 也等于 $W / \tau_{0}$, 其中 $W$ 是一个 ATP 分子水解提供的有效能量, $W-5 \times 10^{-20} \mathrm{~J}^{(9)}, \tau_{0}$ 是一 个 ATP 分子水解提供能量的周期. 由式(1)一-(4)推导得到细胞器转动角速度

$$
\omega=\sqrt{\frac{P}{8 \pi a^{3} \eta}} \text {. }
$$

细胞器旋转角度和转动持续时间 $\boldsymbol{t}$ 的关系式是

$$
\theta=\sqrt{\frac{P}{8 \pi a^{3} \eta}} t \text {. }
$$

设 $a-0.5 \mu \mathrm{m}, \eta=0.075 \times 10^{-1} \mathrm{~Pa} \cdot \mathrm{s}^{[10,11]}$, 若 $\tau_{0}=120 \mathrm{~ms}^{[n}$, 可以求出 $\omega=4 \mathrm{rad} / \mathrm{s}$. 当转动持续时间 $t-0.4 \mathrm{~s}$, 细胞器转动角度 $\theta=92^{\circ}$.

\section{2 细胞器转动与平移运动的比较}

文献[ 7]推导过半径 $\boldsymbol{a}$ 的球形细胞器在粘度 $\boldsymbol{\eta}$ 细胞质中的平移运动. ATP 分子水解提供

1992-02-13㪀稿,1992-10-25收修改稿。 
的驱动功率为 $P$, 细胞器平移运动速度

$$
\nu=\sqrt{\frac{P}{6 \pi a \eta}} .
$$

在运动持续时间 $t$ 内, 平移运动的位移

$$
d=\sqrt{\frac{P}{6 \pi a \eta}} t .
$$

将细胞器转动公式与平移运动公式比较, 可以看到, 转动角速度随球半径的增大而减小 的程度, 比平移运动速度随球半径的增大而减小快得多.

对于同一个细胞器, 如果由 ATP 分子水解提供平移运动的驱动功率和提供转动的驱动 功率相同, 那末当它作平移运动时的平移速度与作转动时的转动角速度之比等于

$$
\frac{\nu}{w}-\frac{2}{\sqrt{3}} a \text {. }
$$

\section{3 细胞器转动和布朗转动}

上面讨论的细胞器由 ATP 分子化学能驱动的转动, 是活细胞内部生命活动的一种表 现. 同时, 分子热运动引起细胞器的转动, 即布朗转动,两者有本质的区别.

文献 [12]给出, 在热平衡条件下, 在温度 $T$ 时, 半径为 $a$ 的球在粘度 $\eta$ 的介质中, 时间间隔 t 内布朗转动角度的均方根

$$
\sqrt{\bar{\Delta}^{2}}-\sqrt{\frac{k T t}{4 \pi a^{3} \eta}}
$$

式中 $k$ 是玻尔兹曼常数. 由式(6),(10), 在相同的时间间隔 动的角度 $\theta$ 与布朗转动角度均方根值 $\sqrt{\overline{\Delta^{2}}}$ 的比值等于

$$
\frac{\theta}{\sqrt{\bar{\Delta}^{2}}}-\sqrt{\frac{E}{2 k T}} \text {. }
$$

如同文献[7]所指出, 由于 $E \gg k T$, 无生命的布朗转动远远小于作为生命活动表现的细胞器 转动. 本文提出的定量关系式, 可以用实验测量来进行检验.

\section{参考文城}

[1] Schliwa, M., in Cell and Muscle Motility, Vol. 5 (ed. Shay, J. W.), Raven Press, New York, 1984.

[2] Rebhum, L. I., Intern. Rev. Cytol., 1972, 32: 93.

[ 3 ] Kachar, B., Science, 1985, 227: 1355.

[ 4 ] Weiss, D. G., Allen, R. D., in Microtubules and Microtubule Inhibitors (eds. De Brabander, M., De Mey, J.)h Elsevier Science Publishers, Amsterdam, 1985, 233.

[5] Williamson, R. E., Plant Physiol, 1986, 82: 631.

[6] Schmitt, F. O., Neurosci. Res. Prog. Bull., 1968, 6: 119.

[7] 度孝威,科学通报, 1992,37(8): 750-752.

[8] Schlichting, H., Boundary-layer Theory, McGraw-Hill Book Company, 1979.

[9] Alberts, B. et al., Molecular Biology of the Cell, 2nd edition, Garland Publishing, Inc., 1989.

[10] Allen, N. S., Jour. Cell Biol., 1974, 63: 270.

[11] Haak, R. A. et al., J. Physiol. (London), 1976, 263: 115.

[12] Einstein, A., Annalen der Physik, 1905, 17: 549. 